\title{
INFLUENCIA DEL ENTRENADOR EN LA DEMANDA TÉCNICA DE UN JUEGO REDUCIDO EN FÚTBOL-7
}

\section{INFLUENCE OF COACH ENCOURAGEMENT ON TECHNICAL DEMAND OF AN SMALL-SIDED GAMES IN FOOTBALL-7}

\author{
Sánchez-Sánchez, J. ${ }^{1}$; Rodríguez, A. ${ }^{2}$; Luis-Pereira, JM. ${ }^{3}$ y Caro, $0 .{ }^{4}$ \\ ${ }^{1}$ Doctor en Ciencias de la Actividad Física y el Deporte. Facultad de Educación. Universidad Pontificia de \\ Salamanca. España. jsanchezsa@upsa.es \\ ${ }^{2}$ Licenciado en Ciencias de la Actividad Física y el Deporte. IBIOMED. Universidad de León. España. \\ arodrf06@estudiantes.unileon.es \\ ${ }^{3}$ Graduado en Ciencias de la Actividad Física y el Deporte. Facultad de Educación. Universidad Pontificia \\ de Salamanca. España. jesuspereira77@gmail.com \\ ${ }^{4}$ Doctor en Ciencias de la Actividad Física y el Deporte. Facultad de Ciencias de la Actividad Física y del \\ Deporte Universidad de Granada. España. NSMP Excellence in Football Project, Aspire ZF. Qatar. \\ oscar.caro10@gmail.com
}

Código UNESCO: 5801 Teoría y Métodos Educativos

Clasificación Consejo de Europa: 17 Otras: Entrenamiento deportivo

Recibido el 30/7/2014

Aceptado el 7/12/2014

\begin{tabular}{l}
\hline PALABRAS \\
CLAVE: \\
Tareas espacio \\
reducido, \\
Entrenador, \\
Entrenamiento \\
técnico, \\
Fútbol \\
formativo
\end{tabular}

KEY WORDS:

Small-Sided

Games,

Coach

encouragement,

Technical

training,

Young football

\section{RESUMEN}

El objetivo del presente estudio ha sido analizar el efecto de la motivación del entrenador sobre la demanda técnica de un juego reducido (JR) 3 contra 3, realizado por futbolistas de categoría alevín, observando el nivel de aprendizaje técnico adquirido a través de estas tareas.

Se han cuantificado el número de acciones técnicas que se producen en un JR de 3 contra 3 sin intervención del entrenador (JRSIE) y las que se producen en el mismo JR con la intervención del entrenador (JRCME). Se ha realizado en cada una de las 4 sesiones del programa de entrenamiento con JR dos test de valoración del dominio técnico en situaciones pre y post sesión.

Los resultados confirman que aparecen diferencias en el número de acciones técnicas que el jugador realiza durante el JR, siendo significativamente mayor en el JRCME, generándose además mayor nivel de aprendizaje.

\section{ABSTRACT}

The aims of this study were to examine the effect of coach encouragement in the technical demand of small-sided games 3-a-side in young football players. In this study were analysed also the level of learning acquired for the players.

The numbers of technical actions doing during small-sided games 3-a sided with and without coach encouragement was counted during every session of the program. Specific technical test was used to identify the learning level acquired. In base of the results of this study, the coach encouragement should consider as an important factor for the SSG design and for the technical training of the young player. 


\section{INTRODUCCIÓN}

El entrenamiento del futbolista durante las etapas de formación puede ser abordado a través de dos perspectivas metodológicas diferenciadas: una más analítica, que utiliza actividades dirigidas al desarrollo de los diferentes contenidos de manera aislada; y otra global, donde a través de una única tarea de entrenamiento se consigue estimular a la vez la mayoría de los factores implicados en el rendimiento deportivo ${ }^{1}$. A pesar de que el fútbol es una de las disciplinas más estudiadas en la literatura científica ${ }^{2}$, no ha sido posible deducir cuál de estas estrategias puede ser la más idónea para conseguir desarrollar el talento de forma óptima en los jóvenes futbolistas ${ }^{3}$. No obstante la complejidad y la elevada cantidad de factores a entrenar en el fútbol moderno, el reducido tiempo de entrenamiento entre competiciones y la importancia del juego colectivo, parecen haber provocado que el entrenamiento evolucione hacia metodologías integrales ${ }^{4}$. En la actualidad la intervención global, basada en la utilización de juegos reducidos $(J R)$ se ha convertido en una forma de entrenamiento muy popular ${ }^{5}$, con un gran seguimiento por parte de entrenadores de deportes como el fútbol ${ }^{6}$. Aunque su aplicación no depende de la edad ó la experiencia previa del jugador ${ }^{7}$, puede ser especialmente interesante aplicarla a jugadores en período de iniciación al deporte ${ }^{8}$. Esto puede ser debido a ciertas ventajas asociadas a este tipo de ejercicios, como el aumento de la motivación que provoca en los participantes, el desarrollo de la conciencia táctica, la mejora de las habilidades técnicas, la optimización del tiempo de práctica y el reducido riesgo de lesión?

Los JR, también conocidos como entrenamiento basado en el juego ${ }^{10}$, son tareas que se realizan en un espacio más pequeño del reglamentario, implicando a un menor número de jugadores, y usando una modificación de las normas oficiales del fútbol- $11^{5}$. Esto permitirá trazar en el entrenamiento objetivos muy concretos, a modo de episodios parciales, que se dirigen a estimular uno o varios planos del rendimiento en fútbol ${ }^{2}$. Además, la mejora de estos factores es altamente específica ${ }^{11,12}$ ya que se incide sobre los aspectos técnicos y tácticos ${ }^{13,14,6}$, sin perder el compromiso fisiológico y la trascendencia competitiva propia de un partido ${ }^{15}$.

En su origen los JR se idearon para que los jugadores pudieran mejorar los elementos técnico-tácticos del juego ${ }^{16,17}$. Sin embargo gran parte de la investigación desarrollada en este ámbito está dirigida al análisis de las respuestas fisiológicas provocadas por este tipo de tareas 3 18,19. El estudio de estas variables es importante pero no suficiente, puesto que el rendimiento en el fútbol también depende del entrenamiento de factores estratégicos, psicológicos, tácticos y técnicos ${ }^{20}$. En un estudio de Di Salvo y cols. ${ }^{21}$, realizado con jugadores profesionales, se observó que en una liga de 20 equipos, los 15 peores clasificados realizaban más actividad de alta intensidad que

Rev. Ib. Cᄃ. Act. Fís. Dep. 
los 5 primeros equipos de la clasificación. Esto nos sugiere que en niveles altos de rendimiento el componente técnico-táctico es determinante. Por este motivo desde las etapas de formación se deben diseñar y proponer tareas que incidan correctamente sobre estos elementos del rendimiento en fútbol.

En la literatura aparecen estudios que han analizado la demanda técnica de JR en función de la intervención sobre las dimensiones del espacio ${ }^{22,23,11}$, la orientación del espacio $^{24}$, el número de jugadores participantes $^{18,25,26}$ o el empleo de jugadores como apoyos externos $^{27,28,29}$. El entrenador debe tener la habilidad para emplear positivamente estos elementos en la construcción de sus medios de entrenamiento, ya que tienen una influencia directa sobre el número de acciones que el jugador realiza, la continuidad de la acción durante el juego o el balance de errores y aciertos $^{28}$. Aunque el área de juego, el número de jugadores y la modificación de las reglas han sido las variables más empleadas en la construcción de JR, otra como la motivación del entrenador también tiene una gran importancia y debe ser considerada para el diseño de la tarea $^{16}$.

En el ámbito del entrenamiento algunos trabajos demuestran que la presencia activa del entrenador durante la ejecución puede incrementar la carga de trabajo ${ }^{30} \mathrm{e}$ incluso la adherencia a la práctica ${ }^{31}$. En referencia al entrenamiento del futbolista a través de JR, algunos estudios han analizado el efecto del aliento del entrenador sobre indicadores físicos ${ }^{32,16,33,34}$, pero hasta donde conocemos no existen trabajos que hayan analizado el efecto de esta variable sobre el comportamiento técnico. Consideramos que este aspecto debe ser controlado en las etapas de formación, donde los factores técnico-tácticos son especialmente interesantes ${ }^{26}$.

\section{OBJETIVOS}

El objetivo del presente estudio ha sido analizar el efecto de la motivación del entrenador sobre la demanda técnica de un juego reducido 3 contra 3 , realizado por futbolistas de categoría alevín, analizando el nivel de aprendizaje técnico adquirido a través de estas tareas.

\section{MATERIAL Y MÉTODOS}

Un grupo de 12 jugadores de fútbol de categoría alevín $(11,33 \pm 0,78$ años de edad; $34,88 \pm 8,39 \mathrm{~kg}$ de peso; $140,75 \pm 0,09 \mathrm{~cm}$ de estatura; 3,00 años de experiencia en el entrenamiento del fútbol) participaron en el estudio. Todos los jugadores realizan 180 minutos de entrenamiento semanal en 2 sesiones, junto a un partido de competición de fútbol-7 los sábados. Tras presentar el correspondiente consentimiento informado completado por los padres, y con el visto bueno del departamento técnico del club, los jugadores desarrollaron el diseño experimental programado, que respeta las condiciones éticas establecidas en la Declaración de Helsinki.

Rev. lb. Cᄃ. Act. Fís. Dep. 
El estudio se realizó durante 2 semanas, con 4 sesiones en total, realizando en la primera sesión de entrenamiento semanal (martes a las 18:00 horas) el registro del peso (Báscula TANITA® MC-418MA "segmental", precisión 100gr) y la talla (Tallímetro Holtex®, precisión 1 $\mathrm{cm})$, y posteriormente el JR sin intervención del entrenador (JRSIE). En la segunda sesión del microciclo (jueves a las 18:00 horas), los mismos futbolistas desarrollaron una tarea idéntica a la realizada en la anterior sesión, pero ahora con la participación activa del entrenador durante la ejecución (JRCME). En ambos casos los jugadores son divididos en 2 grupos, que se mantienen para el JRSIE y para el JRCME. Para evitar el desequilibrio provocado por el nivel futbolístico de los jugadores, los equipos fueron configurados a criterio del técnico del equipo, teniendo en cuenta factores como la demarcación, la competencia técnico-táctica, la aptitud física y la participación en la competición ${ }^{35}$. Todos participan en un JR 3 contra 3 , en un espacio de hierba artificial de $20 \times 15$ metros con mini-porterías (1,5 m de ancho) en los fondos. En la actividad no existe límite de toques, y el objetivo es marcar el mayor número de goles en la portería contraria desde cualquier zona del terreno de juego. Para dar continuidad y evitar en lo posible las interrupciones, se colocan balones fuera del espacio de competición ${ }^{36}$. Los participantes están familiarizados con este tipo de actividades e intervienen con su indumentaria y calzado habitual de entrenamiento. Tanto el JRSIE como el JRCME se aplicaron durante 30 minutos, distribuidos en
6 intervalos de 4 minutos de ejercicio y 90 segundos de recuperación. Durante la pausa los jugadores aprovechan para hidratarse y realizar ejercicios de estiramiento de baja intensidad. Durante el JRSIE el entrenador se limita a marcar los tiempos de cada serie de actividad y arbitrar la tarea, sin poder dirigir ninguna instrucción técnica ni mensajes de ánimo a los participantes. En el JRCME el entrenador además de controlar la actividad, emite mensajes positivos de refuerzo y corrección técnica ("sigue intentándolo"; "bien hecho"; "continúa trabajando así y conseguirás buenos resultados"; "sí colocas bien el pie de apoyo, tu próximo golpeo será más preciso"; "desplázate con el balón mirando a los compañeros" etc.).

Previo al comienzo del JR los futbolistas realizaron 15 minutos de calentamiento, compuesto por 2 minutos de carrera de baja intensidad, $5 \mathrm{~min}$ de movilidad articular, 3 minutos de juego de activación y 5 minutos de acciones técnicas simples por parejas. En cada sesión de evaluación y tras el calentamiento, los jugadores realizan un test de habilidad con balón y otro de pase corto antes (pre-test) y después (post-test) del desarrollo del $\mathrm{JR}^{26}$.

En el test de habilidad con balón ${ }^{37}$ se evalúa la coordinación específica del futbolista. El jugador debe intentar mantener la pelota sin que toque el suelo, utilizando algunas partes de su cuerpo. El entrenador situado a 5 metros del participante, lanzará el balón para que realice la siguiente secuencia: primero, 
pecho, pie y cabeza; segundo, cabeza, pie izquierdo y pie derecho; tercero, pie, pecho y cabeza. El jugador tiene 3 oportunidades cada vez y sumará 1 punto por cada secuencia realizada correctamente.

En el test de pase corto $^{37}$ se evalúa la precisión en el pase. El jugador se desplaza con balón dentro de un rectángulo marcado, y golpea la pelota para intentar meterla dentro de una mini-portería $(1,5 \mathrm{~m}$ de ancho) situada a 11-m de la zona marcada. El jugador realiza una acción con el pie dominante y otra con el no dominante, consiguiendo 3 puntos si el balón entra a gol, 1 punto si da en el poste o travesaño y 0 puntos si sale fuera.

Durante el JR se analizó la intervención del jugador a través del registro de las siguientes acciones técnicas individuales de ataque $y$ defensa: control y pase correcto, pase al primer toque, control y tiro, tiro a primer toque, gol, gol a primer toque, conducción, cabeceo, recuperación de balón y faltas reglamentaria (Tabla 1). Para ello el juego fue filmado con una cámara de vídeo (Sony Handycam HDRPJ240E $®$, SONY China), situada a $10 \mathrm{~m}$ de la zona de juego y sobre una altura de $7 \mathrm{~m}$. Las filmaciones fueron posteriormente manejadas con una herramienta de observación ad hoc, como se ha llevado a cabo en estudios similares ${ }^{22,38,25,11}$. La fiabilidad intraobservador fue evaluada comparando los datos de 2 sesiones de análisis correspondientes a la primera serie de la tarea JRSIE, con un intervalo de separación de 2 semanas ${ }^{35}$. Los resultados muestran alta fiabilidad en la observación con un coeficiente Kappa de Cohen de $0,89^{39}$.

Se calcularon los estadísticos descriptivos (media y desviación estándar) de las variables estudiadas. Tras comprobar la normalidad de la muestra a través de la prueba Saphiro-Wilk, se comparó el valor medio de las acciones técnicas estudiadas en cada grupo de intervención a través de la prueba $t$ Student para muestras independientes. Para comprobar el efecto del JR sobre los test técnicos, se realizó un análisis a través de la prueba $t$ Student para muestras relacionadas. A efectos de comparación de resultados se consideraron diferencias significativas si $p \leq 0,05 \quad\left({ }^{*}\right) \circ p \leq 0,01$ $\left({ }^{* *}\right)$.

Rev. lb. Cᄃ. Act. Fís. Dep. 
Tabla 1. Descripción de acciones técnicas individuales de ataque y defensa analizadas durante la tarea sin intervención (JRSIE) y con intervención activa del entrenador (JRCME).

\begin{tabular}{|c|c|}
\hline Acción & Descripción \\
\hline Control y Pase & $\begin{array}{l}\text { Secuencia de recepción correcta con } \\
\text { dominio del balón, finalizando la acción con } \\
\text { un pase al compañero. }\end{array}$ \\
\hline Pase al primer toque & $\begin{array}{l}\text { El jugador recibe el balón y sin controlar el } \\
\text { mismo, realiza un pase tras un solo } \\
\text { contacto. }\end{array}$ \\
\hline Control y tiro & $\begin{array}{l}\text { Secuencia de recepción correcta con } \\
\text { dominio del balón, finalizando la acción con } \\
\text { un tiro a portería errado. }\end{array}$ \\
\hline Tiro al primer toque & $\begin{array}{l}\text { El jugador recibe el balón y sin controlar el } \\
\text { mismo, realiza un tiro a portería errado tras } \\
\text { un solo contacto. }\end{array}$ \\
\hline Gol & $\begin{array}{l}\text { Cualquier acción de un jugador que, tras } \\
\text { más de un contacto con el balón, finaliza en } \\
\text { gol. }\end{array}$ \\
\hline Gol al primer toque & $\begin{array}{l}\text { El jugador recibe el balón y sin controlar el } \\
\text { mismo realiza un tiro a portería con éxito, } \\
\text { anotando el gol. }\end{array}$ \\
\hline $\begin{array}{l}\text { Conducción y pase } \\
\text { correcto }\end{array}$ & $\begin{array}{l}\text { Secuencia de desplazamiento del jugador } \\
\text { con el balón controlado, finalizando la } \\
\text { acción con un pase recepcionado } \\
\text { adecuadamente por un compañero. }\end{array}$ \\
\hline $\begin{array}{l}\text { Recuperación de } \\
\text { balón }\end{array}$ & $\begin{array}{l}\text { Cualquier acción reglamentaria que permite } \\
\text { a un jugador del equipo en fase defensiva y } \\
\text { sin posesión del balón, controlar el balón } \\
\text { modificando el sentido del juego. }\end{array}$ \\
\hline Cabeceo & $\begin{array}{l}\text { Contacto de la cabeza con el balón en } \\
\text { cualquier momento del juego. }\end{array}$ \\
\hline
\end{tabular}

Rev. Ib. CE. Act. Fís. Dep. 


\section{RESULTADOS}

En la Tabla 2 se muestran los resultados del análisis comparativo de las diferentes categorías de acciones técnicas registradas. Se observa un incremento significativo en el volumen de las ejecuciones en gran parte de los gestos específicos analizados, cuando los futbolistas realizan la tarea con intervención activa del entrenador (JRCME). En comparación con lo ocurrido en el
JRSIE, durante la tarea JRCME se contabilizaron más acciones de control y pase y de tiros al primer toque $(p \leq 0,05)$. También se registraron un mayor número de pases al primer toque, conducción y pase, recuperaciones de balón y de golpeo de balón con la cabeza $(p \leq 0,05)$.

Tabla 2. Frecuencia de acciones técnicas en JR 3 vs. 3 ( $6 \times 4$ min y 90 s recuperación), cuando los jugadores realizan la tarea sin intervención (JRSIE) y con intervención activa del entrenador (JRCME).

\begin{tabular}{lccc}
\hline & JRSIE $(\mathrm{n}=12)$ & JRCME $(\mathrm{n}=12)$ & $\%$ \\
\hline Control y pase correcto & $26,8 \pm 5,03$ & $30,75 \pm 7,84^{*}$ & 14,74 \\
Pase a primer toque & $1,79 \pm 1,02$ & $6,20 \pm 2,84^{* *}$ & 246,37 \\
Control y tiro & $4,79 \pm 2,10$ & $5,16 \pm 1,83$ & 7,72 \\
Tiro al primer toque & $1,87 \pm 1,11$ & $2,79 \pm 1,25^{*}$ & 49,20 \\
Gol & $1,70 \pm 1,85$ & $1,46 \pm 0,72$ & $-14,12$ \\
Gol al primer toque & $0,87 \pm 1,03$ & $1,21 \pm 0,98$ & 39,08 \\
Conducción y pase correcto & $2,17 \pm 1,53$ & $5,41 \pm 3,37 * *$ & 149,31 \\
Recuperación de la posesión & $5,96 \pm 1,68$ & $8,37 \pm 2,67^{* *}$ & 40,44 \\
Cabeceo & $0,58 \pm 0,77$ & $1,33 \pm 0,96 * *$ & 129,31
\end{tabular}

$\%$, Incremento de acciones técnicas en JRCME respecto a JRSIE. Valores medios \pm Desviación típica. "=Diferencias significativas entre JRSIE y JRCME; Niveles de Significación " $=p \leq 0,05 ; "=p \leq 0,01 . n=$ tamaño de muestra.

En la Figura 1 se representan los datos de frecuencia por series de trabajo, de las acciones técnicas de control y pase (a), pase a primer toque (b), tiro al primer toque (c) y recuperación (d). En cuanto a los pases al primer toque, a medida que se acumulan las series de trabajo, se observa una tendencia a disminuir la frecuencia de esta acción. Por otra parte, existe una diferencia significativa $(p \leq 0,05)$ a favor del JRCME en cinco de los seis intervalos de esfuerzo. Con respecto a las recuperaciones, también se observa una tendencia a la reducción de este tipo de acciones en el JRCME a medida que avanza el tiempo de tarea. En las tres primeras series se ha registrado un mayor número de recuperaciones en la tarea realizada con la intervención activa del entrenador $(p \leq 0,05)$.

Rev. lb. Cᄃ. Act. Fís. Dep. 

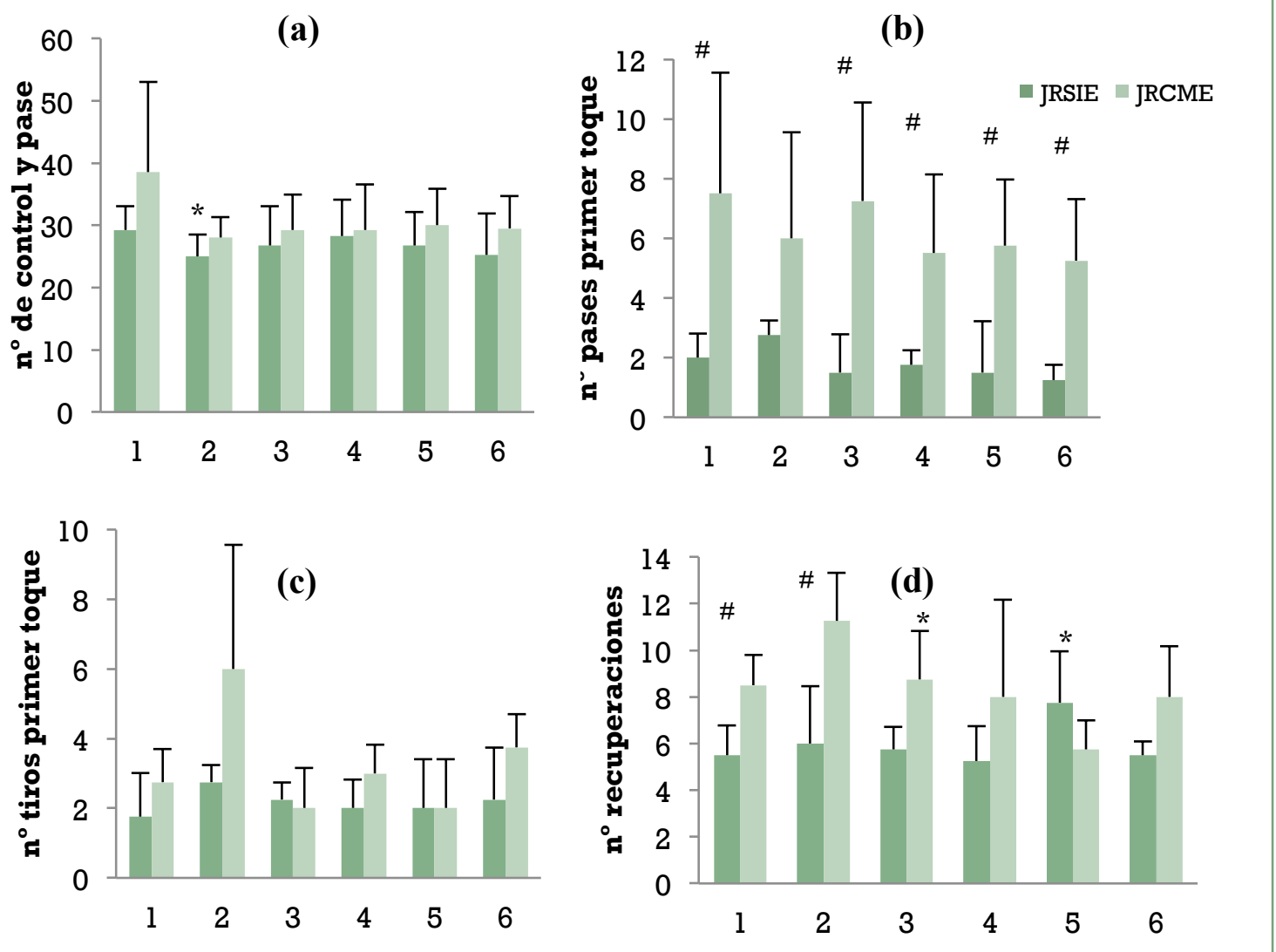

Figura 1. Datos de frecuencia por series de trabajo, de las acciones técnicas de control y pase (a), pase al primer toque (b), tiro al primer toque (c) y recuperación (d). * $p \leq 0,05$ diferencia significativa con respecto al intervalo anterior; ${ }^{*} p \leq 0,05$ diferencia en el número de acciones por intervalo entre el JR sin intervención (JRSIE) y con intervención activa del entrenador (JRCME)

Con respecto a los test técnicos utilizados antes y después de la tarea, en la Figura 2 se puede observar que aparecen diferencias significativas en el nivel obtenido en la prueba de habilidad con balón tras el JRCME, no apareciendo cambios en los niveles de los otros dos test. No obstante, en la mayoría de los casos existe una tendencia a mejorar en el post-test los resultados obtenidos en la prueba antes de la realización de la tarea.

Rev. Ib. Cᄃ. Act. Fís. Dep. 


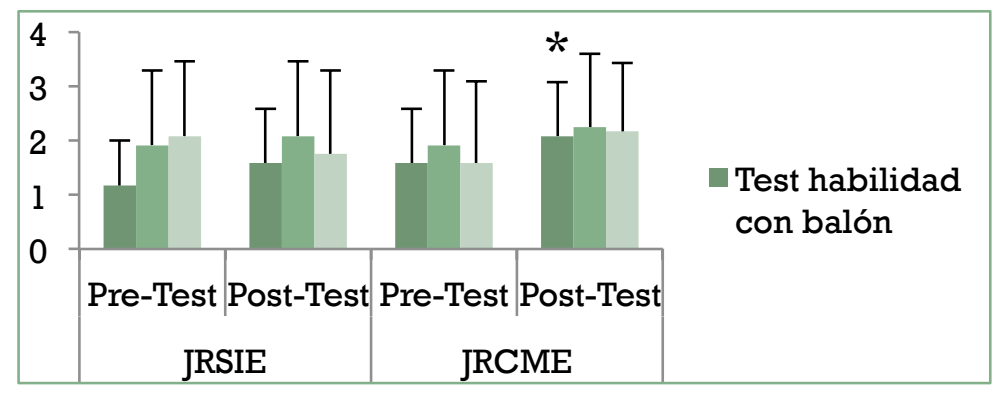

Figura 2. Resultados en los test técnicos antes (pre-test) y después (post-test) de la realización del JR (3 vs. 3, 6x4min y 90s recuperación) sin intervención (JRSIE) y con intervención activa del entrenador (JRCME).

${ }^{*} p \leq 0,05$, diferencia significativa entre resultados del pre-test y post-test.

\section{DISCUSIÓN}

El objetivo del estudio ha sido analizar el efecto de la participación activa del entrenador sobre la demanda técnica de un juego reducido 3 contra 3 , realizado por futbolistas de categoría alevín. Tal y como se puede observar en los resultados obtenidos, en la tarea JRCME se generan un mayor número de acciones técnicas (pase al primer toque, tiro al primer toque, conducción de balón, recuperación y cabeceo) en comparación con el JRSIE. A mayor número de intervenciones del jugador sobre el balón, mayor será el aprendizaje técnico conseguido ${ }^{40,41}$, por lo que los resultados obtenidos pueden tener una importancia capital en relación al análisis del proceso de entrenamiento en el fútbol formativo. Aunque tradicionalmente el entrenamiento de las acciones técnicas se abordaba a través de estrategias predominantemente analíticas $^{1}$, los estudios realizados en la última década en el marco de los JR, han demostrado que estas tareas permiten una elevada intervención del jugador sobre el balón ${ }^{11}$ y con ello la posibilidad de mejorar la ejecución de los gestos técnicos, generándose el aprendizaje en un contexto de alta especificidad $^{2}$.

La información aportada por las distintas investigaciones precedentes sugiere que los JR pueden ser un seguro, efectivo y específico método de entrenamiento en fútbol ${ }^{38}$, ya que se consigue desarrollar, de manera global, la habilidad técnico-táctica del jugador $^{25}$. Para ello es necesario diseñar los JR de una forma concreta para que reúnan una serie de condiciones, que en el marco del entrenamiento técnico, permitan incrementar la intervención del jugador sobre el balón. Algunos estudios precedentes han apuntado que la manipulación del espacio de juego y del número de jugadores (y por tanto del espacio de interacción individual), así como de determinadas reglas propias de la tarea, pueden alteran la demanda de la misma, tanto en relación a la exigencia condicional como técnicotáctica $^{5,9,42}$.

Rev. Ib. Cᄃ. Act. Fís. Dep. 
Hasta el momento, todas las investigaciones que han analizado la influencia del entrenador en los JR han tenido como objeto de estudio el aspecto condicional de la tarea. Se ha demostrado que la participación activa del entrenador aumenta la intensidad, y por tanto, la carga de la tarea, entendida desde el punto de vista fisiológico $^{16,33}$. Como consecuencia de ello, los JR son empleados de forma muy extendida para mejorar determinados aspectos físicos del jugador $^{3}$.

Este aumento de la exigencia condicional podría surgir de la motivación que genera en el jugador la presencia activa del entrenador, que se materializa en una mayor participación de los jugadores ${ }^{3}$. Si bien no ha sido analizado hasta el momento en estudios precedentes, podemos considerar que este aumento de participación es global, ya que no se limita al número de acciones físicas realizadas, sino que aumenta también el número de contactos con el balón. En base a los resultados del presente estudio podemos confirmar que en la tarea desarrollada con la intervención del entrenador se aumentan considerablemente los elementos relacionados con la ejecución del gesto técnico deportivo en relación al balón. Además, se puede comprobar igualmente, que el significativo aumento de las acciones técnicas durante el JRCME, corresponde con aquellas que se pueden asociar con situaciones de juego intensas y veloces, donde el jugador tiene una exigencia espacio-temporal elevada.
Si bien el análisis cuantitativo de los JR que se han desarrollado en este estudio es determinante para dar respuesta al objetivo de la investigación, la riqueza de este trabajo no realizado anteriormente, también reside en la posibilidad de establecer nuevas líneas de investigación, que confirmen el uso de los JRCME en edades formativas, como una herramienta eficaz para mejorar el dominio de los aspectos técnicos del jugador. Para ello, es necesaria la participación del entrenador como impulsor de comportamientos motrices de alto valor para la formación del joven futbolista.

Los test de evaluación técnica empleados en este estudio, están científicamente validados y han sido aplicados en otros diseños que emplean situaciones pre y post test dentro de una sesión de entrenamiento ${ }^{37}$. Se puede observar en los resultados obtenidos una tendencia a la mejora en el posttest. En relación al test de habilidad, en el cual se incluyen una variedad de acciones mayor que en los otros test, se obtienen diferencias significativas a nivel $p \leq 0,05$ en la mejora de los jugadores que realizan el JRCME. Estas diferencias no son significativas en el caso de los jugadores que realizan el JRSIE. Es necesario aumentar el número de sesiones del programa de entrenamiento, para comprobar si el efecto de aprendizaje aumenta de forma significativa. No obstante, en base a los resultados del presente estudio, se observa una tendencia positiva a la presencia del entrenador dentro del JR.

Rev. Ib. CL. Act. Fís. Dep. 


\section{CONCLUSIONES}

Las conclusiones que se pueden obtener del presente estudio son las siguientes:

- La participación activa del entrenador durante los JR provoca un aumento significativo del número de acciones técnicas realizadas por el jugador, en comparación con las que realiza en la misma tarea sin intervención del técnico.

- El aprendizaje de las habilidades técnicas será mayor cuando el jugador esté expuesto a un mayor número de eventos que demanden dicha habilidad. Los JRCME aumentan el número de acciones desarrolladas, por lo que se convierten en ideales dentro de los programas de entrenamiento en la etapa de fútbol base.

- Los JRCME generan situaciones de juego de mayor intensidad. Esta intensidad se traduce en una mayor carga condicional de la tarea, generándose al mismo tiempo un mayor número de acciones técnicas, determinadas por una mayor exigencia espacio-temporal sobre el jugador.

- En futuros estudios se debería aumentar el número de sesiones del programa de entrenamiento, con el objetivo de confirmar, a través de los test aplicados en el presente trabajo, que se genera un aprendizaje significativamente mayor en los jugadores que realizan el JRCME, confirmándose los indicios que aparecen en los resultados de este trabajo.

\section{REFERENCIAS BIBLIOGRÁFICAS}

1. Robles J, Giménez FJ, Abad MT. Metodología utilizada en la enseñanza de los contenidos deportivos durante la ESO. Rev. Int. Med. Cienc. Act. Fís. Deporte. 2011; 11(41): 35-57.

2. Sánchez-Sánchez J, Yagüe JM, Fernández RC, Petisco C. Efectos de un entrenamiento con juegos reducidos sobre la técnica y la condición fisica de jóvenes futbolistas. RICYDE Revista Internacional de Ciencias Del Deporte. 2014; 37: 221-234.

3. Aguiar M, Botelho G, Lago C, Maças V, Sampaio J. A review on the effects of soccer small-sided games. J Hum Kinet. 2012; 33: 103-113.

4. Reilly T. An ergonomics model of the soccer training process. J Sports Scis. 2005: 23(6); 561-72.

5. Hill-Haas SV, Dawson B, Impellizzeri FM, Coutts AJ. Physiology of smallsided games training in football: a systematic review. Sports Med. 2011; 41(3): 199-220.

6. Owen A, Wong P, Paul D, Dellal A. Effects of a periodized small-sided game training intervention on physical performance in elite professional soccer. J Strength Cond Res. 2012; 26(10): 2748-2754.

7. Randers MB, Nybo L, Petersen J, Nielsen JJ, Christiansen L, Bendiksen M, Krustrup P. Activity profile and physiological response to football training for untrained males and females, elderly and youngsters: influence of the number of players.

Rev. Ib. CL. Act. Fís. Dep. 
Scand J Med Sci in Sports. 2010; 1: 14-23.

8. Impellizzeri FM, Marcora SM, Castagna C, Reilly T, Sassi A, laia FM, Rampinini E. Physiological and performance effects of generic versus specific aerobic training in soccer players. Int J Sports Med. 2006; 27(6): 483-92.

9. Little T. Optimizing the Use of Soccer Drills for Physiological Development. J Strength Cond Res. 2009; 31(3): 6774.

10. Gabbett TJ. Skill-based conditioning games as an alternative to traditional conditioning for rugby league players. J Strength Cond Res. 2006; 20(2): 30915.

11. Owen A, Twist C, Ford P. Small-sided games: the physiological and technical effects of altering pitch size and player numbers. Insight FACA J. 2004; 7(2): 50-53.

12. Fradua L, Zubillaga $A$, Caro $O$, Fernandez-García A, Ruiz-Ruiz C, Tenga A. Designing small-sided games for training tactical aspects in soccer: Extrapolating pitch sizes from full-size professional matches. J Sports Sci. 2012; 31(6): 573-581.

13. Dellal A, Chamari K, Pintus A, Girard O, Cotte T, Keller D. Heart rate responses during small-sided games and short intermittenet running training in elite soccer players: a comparative study. J Strength Cond Res. 2009; 22(5): 14491457.

14. Hill-Haas SV, Coutts AJ, Rowsell, GJ, Dawson, BT. Generic versus smallsided game training in soccer. Int $\mathrm{J}$ Sports Med. 2009; 30(9): 636-42.

15. Gabbett T, Jenkins D, Abernethy B. Game-Based Training for Improving Skill and Physical Fitness in Team Sport Athletes. Int J Sports Sci Coach. 2009; 4(2): 273-283.

16. Rampinini E, Impellizzeri FM, Castagna C, Abt G, Chamari K, Sassi A, Marcora SM Factors influencing physiological responses to small-sided soccer games. J Sports Sci. (2007); 25: 659666.

17. Reilly T, Gilbourne D. Science and football: a review of applied research in the football codes. J Sports Scis.. 2003; 21(9): 693-705.

Rev. Ib. Cᄃ. Act. Fís. Dep.
18. Hill-Haas SV, Dawson BT, Coutts AJ, Rowsell GJ. Physiological responses and time-motion characteristics of various small-sided soccer games in youth players. J Sports Scis. 2009; 27(1): 1-8.

19. Hoff J, Wisloff U, Engen LC, Kemi OJ, Helgerud J. Soccer specific aerobic endurance training. $\mathrm{Br} \mathrm{J}$ Sports Med. 2002; 36: 218-221.

20. Stølen T, Chamari K, Castagna C, Wisløff U. Physiology of Soccer. Sports Med. 2005; 35(6): 501-536.

21. Di Salvo V, Gregson W, Atkinson G, Tordoff P \& Drust B. Analysis of high intensity activity in premier league soccer. Int Jof Sports Med. 2009; 30: 205-212.

22. Casamichana D, Castellano J. Timemotion, heart rate, perceptual and motor behaviour demands in smallsides soccer games: effects of pitch size. J Sports Scis. 2010; 28(14): 1615-23.

23. Kelly DM, Drust B. (2009). The effect of pitch dimensions on heart rate responses and technical demands of small-sided soccer games in elite players. J Sci Med Sport. 2009; 12(4): 475-9.

24. Mallo J, Navarro E. Physical load imposed on soccer players during small-sided training games. 2008; 48(2): 166-171,

25. Jones S, Drust B. Physiological and technical demands of $4 \vee 4$ and $8 \vee 8$ games in elite youth soccer players. Kinesiology. 2007; 39(2): 150-156.

26. Katis A, Kellis E. Effects of small-sided games on physical conditioning and performance in young soccer players. $J$ Sports Sci Med. 2009; 8: 374-380.

27. Dellal A, Hill-Haas S, Lago-Penas C, Chamari K. Small-sided games in soccer: amateur vs. professional players's physiological responses, physical, and technical activities. J Strength Cond Res. 2011; 25(9): 23712381.

28. Dellal A, Lago-Penas C, Wong DP, Chamari K. Effect of the number of ball contacts within bouts of 4 vs. 4 smallsided soccer games. Int J Sports Physiol. Perform. 2011; 6(3): 322-33.

29. Dellal A, Chamari K, Owen AL, Wong DP, Lago-Penas C, Hill-Haas S. Influence of technical instructions on 
the physiological and physical demands of small-sided soccer games. Eur J Sport Sci. 2011; 11(5): 341-346.

30. Mazzetti SA, Kraemer WJ, Volek JS, Duncan ND, Ratamess NA, Gómez AL, Fleck SJ. The influence of direct supervision of resistance training on strength performance. Med Sci Sports Exerc. 2000; 32(6): 1175-84.

31. Coutts AJ, Murphy AJ, Dascombe, BJ. Effect of direct supervision of a strength coach on measures of muscular strength and power in young rugby league players. J Strength Cond Res. 2004; 18(2): 316-323.

32. Balsom P. Precision Football. Kempele; Finland: 1999.

33. Sampaio J, Garcia G, Macxas V, Ibanez SJ, Abrantes C, Caixinha P. Heart rate and perceptual responses to $2 \times 2$ and $3 \times 3$ small-sided youth soccer games. JSSM. 2007; 6 (10): 121-122.

34. Sassi R, Reilly $T$, Impellizzeri FA. Comparison of small sided games and interval training in elite profesional soccer players. J Sports Sci. 2004; 22: 562.

35. Casamichana D, Castellano J. Análisis de los diferetnes espacios individuales de interacción y los efectos en las conductas motrices de los jugadores: aplicaciones al entrenamiento en futbol. Mot Eur J Hum Mov. 2009; 23: 143167.

36. Köklü $Y$, Aşçi A, Koçak FU, Alemdaroğlu U, Dündar U. Comparison of the physiological responses to different small-sided games in elite young soccer players. J Strength Cond Res. 2011; 25(6): 15221528.

37. Rösch D, Hodgson R, Peterson L, GrafBAumann T, Junge A, Chomiak J, Dvorak J. Assessment and evaluation of football performance. Am Jof Sports Med. 2000; 28(5): 29-39.

38. Gabbett TJ, Mulvey MJ. Time-motion analysis of small-sided training games and competition in elite women soccer players. J Strength Cond Res. 2008; 22(2): 543-552.

39. Cohen J. Statistical Power Analysis for the Behavioral Sciences. Hillsdale, NJ: Lawrence Erlbaum Associate. 1988.

40. Mendez Giménez A. Modelos de enseñanza deportiva. Análisis de dos décadas de investigación. Ef Deportes. 1999; 13.

41. Wein H. Fútbol a la medida del niño. 1. Madrid: Gymnos; 2004.

42. Caro O. Análisis de los espacios de juego en fútbol profesional y su extrapolación al diseño de las tareas de entrenamiento. Tesis Doctoral (no publicada). 2014. Granada.

Rev. Ib. Cᄃ. Act. Fís. Dep. 